NSF-ITP-97-019 gr-qc/9703027

\title{
Black Hole Boundary Conditions and Coordinate Conditions
}

\author{
Douglas M. Eardley* \\ Institute for Theoretical Physics \\ University of California \\ Santa Barbara, CA 93106-4030
}

(July 2, 2021)

\begin{abstract}
This paper treats boundary conditions on black hole horizons for the full $3+1 \mathrm{D}$ Einstein equations. Following a number of authors, the apparent horizon is employed as the inner boundary on a space slice. It is emphasized that a further condition is necessary for the system to be well posed; the "prescribed curvature conditions" are therefore proposed to complete the coordinate conditions at the black hole. These conditions lead to a system of two 2D elliptic differential equations on the inner boundary surface, which coexist nicely to the $3 \mathrm{D}$ equation for maximal slicing (or related slicing conditions). The overall $2 \mathrm{D} / 3 \mathrm{D}$ system is argued to be well posed and globally well behaved. The importance of "boundary conditions without boundary values" is emphasized. This paper is the first of a series.
\end{abstract}

*Electronic address: doug@itp.ucsb.edu 


\section{INTRODUCTION}

If we need to define a flat surface, an elastic membrane — such as a soap bubble stretched over a fixed rigid ring does a good job. The shape of the membrane is governed by the Laplace equation, with Dirichlet boundary conditions at its edge determined by the ring. What if we additionally need the membrane to have a hole somewhere near its center? If there is a small rigid ring available, we can simply drop it onto the membrane, and then cut out a hole in the membrane, fixing the new, inner edge to the small ring, again with Dirichlet boundary conditions. Overall, the small ring floats according to forces from the membrane, but the ring determines the size and shape of the hole. What do we do if no suitable small rigid ring is available? Our metaphor, admittedly loose, of course pertains to the study of black holes by numerical relativity, where indeed there is no suitable rigid "ring".

If we have closed loop of elastic string, it can serve as the inner boundary. The size and shape of the hole is not fixed, but rather determined by force balance between the string and the membrane. We may guess the equilibrium shape of the hole to be a circle. The curvature of the circle, and hence the size of the hole, will be determined by balance between tension along the string and surface tension in the membrane. The equilibrium will be described by some differential equations: In the membrane, the 2D Laplace equation; and along the string, two stationary wave equations - two, because the string has two transverse degrees of freedom in space. [ Boundary conditions at the edge of the hole couple all of these equations together.

Let us now pass from the metaphorical soap bubble to the real black hole. Relativists have long hoped to pose numerical boundary conditions at the horizon of a black hole, and use the horizon as the inner boundary of the numerical grid. Recently, progress has been achieved in spherical symmetry by Scheel, Shapiro \& Teukolsky [1],2], and by Anninos, Daues, Masso, Seidel, and Suen [3] (see also [4]). These two groups carried out important demonstrations of the feasibility of such horizon boundary conditions by evolving spherically symmetric black holes (in Brans-Dicke theory and Einstein theory respectively). The basic idea is to make the inner boundary an apparent horizon.

It would be highly desirable to extend such horizon boundary conditions to more complex situations involving distorted or rotating black holes, and black hole binaries. The main purpose of this paper is to work out the underlying theory of these coordinate conditions in 3D generality, as a step of such an extension. In particular the 2D differential equations governing such inner boundary surfaces will be studied with a view toward making them well-posed, when connected to the 3D equation for maximal slicing in relativity, or to various other slicing conditions (i.e., choice of lapse) such as generalizations of maximal slicing [5]. The basic idea of using an apparent horizon will turn out to work, but several difficult issues crop up along the way, and must be dealt with. We need boundary conditions at the horizon, but we do not know how to supply boundary values there; how to resolve this issue of "boundary conditions without boundary values" is explained along the way.

\footnotetext{
${ }^{1}$ The real string also has a longitudinal degree of freedom; but this can be decoupled by attaching the membrane to the string with a slip boundary — as with a soap bubble.
} 
The development in this paper will not make specific assumptions about the conditions for 3+1D slicing and 3D spatial coordinates, though we will often assume that these conditions are implemented by some kind of 3D elliptic or parabolic equations, which will require boundary conditions at the hole. Hyperbolic operators (e.g., arising in harmonic slicing or harmonic coordinates) form quite a different case, and probably a simpler one, since they do not require boundary conditions at the hole. (I am grateful to Greg Cook for pointing this out.)

In future papers in this series, further topics will be treated:

- Horizon boundary conditions for stationary black holes, relevant to the late stages of numerical calculations.

- The connection between spatial coordinate conditions, i.e., choice of shift, and horizon boundary conditions; this is especially important for rotating black holes.

- Boundary conditions for the constraint equations of general relativity on horizons; again a key issue is "boundary conditions without boundary values".

\section{MOST APPARENT HORIZONS ARE WILD}

Hawking [6] defined trapped surfaces and apparent horizon as follows.

Definition 1. A trapped surface $S$ is an achronal 2-surface in spacetime for which the outward null convergence obeys

$$
\rho>0
$$

A marginally trapped surface $S$ is one for which the outward null convergence obeys

$$
\rho=0
$$

The basic property of these surface is:

Proposition 2. (Hawking \& Ellis [6]). Given a trapped or marginally trapped surface $S$. No point $p$ on $S$ can lie outside the event horizon.

Definition 3. In a given space slice $V$, an apparent horizon is the outward boundary of trapped surfaces that lie in $V$.

Notice that the given slice $V$ is an essential ingredient of the definition. To say that "We are going to choose a slice that meets an apparent horizon..." is a circular definition. This issue is not just a niceity; to ignore it can lead to serious trouble, as we will soon see. One way to see the problem is to observe that any slice passing far enough into the black hole over a wide range - will meet an apparent horizon, and therefore will obey the boundary condition. A condition that excludes nothing is not a useful condition.

\footnotetext{
${ }^{2}$ On the other hand, it is not clear whether harmonic slicing avoids coordinate singularities.
} 
To de-circularize the definitions, we must drop the slice, and refer not to an apparent horizon, but to a marginally trapped surface. So now we can sensibly say "We are going to choose a slice that meets a marginally trapped surface." And we can proceed to build a $3+1 \mathrm{D}$ code that uses marginally trapped surfaces $S$ as the inner boundary of the slices. However, this code may not work; it is vulnerable to crashing after a short time. The pitfall is that "most" marginally trapped surfaces are wild surfaces; this is very unlike the familiar situation with maximal slices, which are automatically smooth (technically, thanks to elliptic regularity). The wildness of marginally trapped surfaces is intrinsically a non-sphericallysymmetric phenomenon, and does not show up at all in spherically symmetric setting consistent with the success of Anninos, Daues, Masso, Seidel, and Suen.

A related property of a generic marginally trapped surface, is that it can always be deformed spatially outward from any point on it:

Proposition 4. Given any smooth marginally trapped surface $S$, such that either $\sigma$ or $T_{\mu \nu} l^{\mu} l^{\nu}$ does not vanish identically on $S$. Given any point $p$ on $S$, and given a spacelike outward-pointing vector $u$ at $p$. Then $S$ can be locally perturbed into a 1-parameter family of marginally trapped surfaces $S(\epsilon)$ so that $p$ moves in the u-direction.

(Here $\sigma$ is the outward null shear, $T_{\mu \nu} l^{\mu} l^{\nu}$ is the outward null component of the stress energy tensor, and we will assume throughout that $T_{\mu \nu} l^{\mu} l^{\nu}$ obeys the dominant energy condition. Actually Proposition 4 is true for any $u$, but is most interesting if $u$ is spacelike outward-pointing.)

This leads to a puzzle. Start with an apparent horizon $S$ and some point $p$ on it. What is to keep us from continually perturbing it, extending the 1-parameter family $S(\epsilon)$, until $p(\epsilon)$ passes outside the event horizon, contradicting Proposition 2? The only thing that can go wrong is that $S$ must "go wild" - i.e., cease being smooth - first.

In fact, the following conjecture, at first a bit startling, can be surmised as the obvious general answer to the puzzle.

Conjecture A. The outward boundary in spacetime of marginally trapped surfaces is the event horizon.

I have proven this conjecture under some assumptions, plausible but not rigorously established, about how spacetime settles down to a nonextremal black hole subsequent to gravitational collapse. The trapped surfaces $S$ that pass close to the event horizon are wild. Let us give an example to illustrate what happens.

Sometime during the dynamical phase of collapse, after the black hole has formed but before it has settled down, choose a slice that contains an apparent horizon $S$, and choose a point $p$ on $S$. Also choose any point $q$ on the intersection of the slice with event horizon. In the usual picture, $q$ is considerably outside the apparent horizon, so it is unclear how any trapped surface can pass close to $q$.) However, by Proposition 4, we can now extend a 1-parameter family of marginally trapped surfaces $S(\epsilon)$, so that $p(\epsilon)$ remains in the slice $V$ (though most of $S(\epsilon)$ does not) and so that $p(\epsilon) \rightarrow q$ at some parameter value $\epsilon_{0}$. What happens is that all of $S(\epsilon)$ approaches the event horizon as $\epsilon \rightarrow \epsilon_{0}$, but that most of $S(\epsilon)$ moves far to the future. Therefore the trapped surfaces $S$ which pass close to $q$ look as follows. Most of $S$ lies far to the future, very close to the event horizon of the settled-down black hole. Only a thin tendril of $S$ extends back near $q$, in a thin tubular neighborhood 
of a generator of the event horizon. Though this tendril is nearly null, it is still part of a spacelike surface.

Let us see what this means for numerical relativity. Each marginally trapped surface $S(\epsilon)$ can serve as the inner boundary of a slice $V(\epsilon)$, say a maximal slice, extending to spatial infinity. Moreover we can arrange for $\epsilon$ to be proper time at infinity, so that the slicing "goes wild" at finite time. This is the serious trouble that such a code can run into.

Details will be published elsewhere.

\section{THE PRESCRIBED CONVERGENCE CONDITIONS FOR 2-SURFACES}

This is not to say that apparent horizons are necessarily a bad idea for inner boundary conditions, only that an ingredient is missing. We propose that the missing ingredient is the simplest possible thing, the convergence $\rho^{\prime}$ of the inward null normal to a surface $S$, and that the well posed way to put an inner boundary on a slice is to prescribe both the outward null convergence $\rho$ and the inward null convergence $\rho^{\prime}$.

\section{A. The Two Mean Extrinsic Curvatures of a 2-Surface in Spacetime}

Consider a spatial 2-surface $S$ immersed in spacetime. (Throughout, $S$ will be topologically a 2-sphere unless otherwise noted.) All over $S$, we can choose an orthonormal frame of reference $\mathbf{e}_{\hat{\alpha}}$ so that the time axis $\mathbf{e}_{\hat{0}}$ and one of the spatial axes $\mathbf{e}_{\hat{1}}$ are normal to $S$, while the other two spatial axes $\mathbf{e}_{\hat{2}}, \mathbf{e}_{\hat{3}}$ are tangent to $S$. Then $S$ has defined on it two mean extrinsic curvatures, namely $H_{0}$ in the $\hat{0}$-direction, and $H_{\hat{1}}$ in the $\hat{1}$-direction. If $\sqrt{h}$ is the element of area on $S$, then in suitable local coordinates we can take as the definitions

$$
\begin{aligned}
& \partial_{\hat{0}} \sqrt{h}=-H_{\hat{0}} \sqrt{h} \\
& \partial_{\hat{1}} \sqrt{h}=-H_{\hat{1}} \sqrt{h}
\end{aligned}
$$

(the minus sign in these equations is a matter of convention). In any coordinate system, these mean extrinsic curvatures form a 4-vector $H_{\mu}$ orthogonal to $S$.

Alternatively we can follow null methodology [7] and choose two orthonormal null vectors, the outward and inward null normals to $S$ respectively, as

$$
\begin{array}{r}
\mathbf{l}=\frac{\mathbf{e}_{\hat{0}}+\mathbf{e}_{\hat{1}}}{\sqrt{2}} \\
\mathbf{n}=\frac{\mathbf{e}_{\hat{0}}-\mathbf{e}_{\hat{1}}}{\sqrt{2}}
\end{array}
$$

with $l_{\mu} l^{\mu}=0=n_{\mu} n^{\mu}, l_{\mu} n^{\mu}=-1$, and then the null convergences of $\mathbf{l}$ and $\mathbf{n}$ are

$$
\begin{array}{r}
\rho=\frac{H_{\hat{0}}+H_{\hat{1}}}{\sqrt{2}} \\
\rho^{\prime}=\frac{H_{\hat{0}}-H_{\hat{1}}}{\sqrt{2}}
\end{array}
$$


Notice, however, that we had to choose some frame of reference on $S$ to define $H_{\hat{0}}$ and $H_{\hat{1}}\left(\right.$ or $\rho$ and $\rho^{\prime}$ ). The arbitrariness in choice of frame amounts to a boost in the 1-direction (a GHP boost) all over $S$ [7]. Under such a boost these quantities transform like

$$
\begin{aligned}
\mathbf{l} \rightarrow \tilde{\mathbf{l}} & =e^{\psi} \mathbf{l} \\
\mathbf{n} \rightarrow \tilde{\mathbf{n}} & =e^{-\psi} \mathbf{n} \\
\rho & \rightarrow \tilde{\rho}=e^{\psi} \rho \\
\rho^{\prime} \rightarrow \tilde{\rho}^{\prime} & =e^{-\psi} \rho^{\prime}
\end{aligned}
$$

where the boost rapidity $\psi$ is an arbitrary function on $S$. Only one boost-invariant scalar can be formed from the mean extrinsic curvatures, namely $H_{\mu} H^{\mu}=-H_{\hat{0}}^{2}+H_{\hat{1}}^{2}=-2 \rho \rho^{\prime}$.

How then do we determine a 2 -surface $S$ in spacetime? Since $S$ has two transverse degrees of freedom, we need two conditions at each point of $S$, that is, two equations in two coordinates. It is tempting therefore to put conditions on the two quantities $\left\{H_{\hat{0}}, H_{\hat{1}}\right\}$ or equivalently $\left\{\rho, \rho^{\prime}\right\}$ :

$$
\begin{array}{r}
\rho=(\text { some fixed function on } S) \\
\rho^{\prime}=(\text { some other fixed function on } S)
\end{array}
$$

(For instance, to use an apparent horizon we can just take $\rho=0$ as one of the two conditions.) These equations will be called the equations of prescribed convergence, or, for short, the $P C$ equations. However the PC equations are not enough by themselves, because of the boost arbitrariness, Eq. (6). We are missing one condition, namely something to fix $\psi$ as a function of two coordinates on $S$.

If we have already decided on a slicing condition for spacetime, e.g., maximal slicing, or something related to it, then the slice itself provides the missing condition: We can use the unit timelike normal to the slice to fix the $\hat{0}$-direction at $S$. Then the PC equations, together with the maximal slicing equation, are just enough. This is of course no guarantee that the equations form a well-posed system, meaning that $V$ and $S$ can never "go wild". However it is a reasonable conjecture that this is so, and this paper will present a considerable amount of evidence in favor of this conjecture.

\section{B. The $3+1$ and $2+1$ Splits}

The spacetime metric is

$$
d s^{2}=-\left(\alpha^{2}-\beta_{i} \beta^{i}\right) d t^{2}+2 \beta_{i} d x^{i} d t+\gamma_{i j} d x^{i} d x^{j}
$$

The notation is now necessarily going to become a little complicated, so the reader is asked to be patient. Starting from the full spacetime geometry, we choose slices and carry out the standard $3+1$ split. The unit future-pointing timelike normal to the slices is denoted $\mathbf{e}_{\hat{0}}$. Spacetime indices run over $\mu, \nu, \ldots=0,1,2,3$, where 0 denotes time $t$; spatial indices run over $i, j, \ldots=1,2,3$, and can be lowered and raised with the spatial metric $\gamma_{i j}$ and its inverse $\gamma^{i j}$.

Each space slice $t=$ const has an inner boundary on a 2 -surface $S$. For convenience in this paper, we will throughout choose spatial coordinates $\left\{x^{i}\right\}$ and shift vector $\beta^{i}$ so that 


$$
S \text { always lies at } r=r_{0}=\text { const, where } r \equiv x^{1} \text {; }
$$

This means that $\beta_{i}$ is not freely specifiable at $S$, but must be chosen to match the motion of $S$ :

$$
B \equiv \beta_{i} e_{\hat{1}}^{i} \quad \text { fixed at } S \text { by the PC conditions }
$$

here $e_{\hat{1}}^{i}$ is the unit outward spatial normal to $S$. One would actually like more general spatial coordinates, to allow the black hole to move through the spatial coordinate system - to "fly through the grid". The generalization to such coordinates is straightforward but is not developed in this paper.

The Riemannian 2-metric on $S$ will be denoted $h_{a b}$; surface indices run over $a, b, \ldots=2,3$ and can be lowered and raised with $h_{a b}$ and its inverse $h^{a b}$. We use a $2+1$ split at $S$, and 2 -tensors on $S$ will carry indices $a, b, \ldots$. The spatial extrinsic 2-curvature of $S$ with respect to the spatial normal direction $e_{\hat{1}}^{i}$ is a 2 -tensor

$$
H_{a b} \equiv-\frac{1}{2} \mathcal{L}_{\hat{1}} h_{a b}
$$

where $\mathcal{L}$ denotes Lie derivative, and the spatial mean extrinsic 2-curvature $H_{\hat{1}}$ is its 2-trace,

$$
H_{\hat{1}}=h^{a b} H_{a b}=-\frac{1}{\sqrt{h}} \partial_{\hat{1}} \sqrt{h}
$$

where $h \equiv \operatorname{det} h_{a b}$. The Gauss-Codazzi equations of the $2+1$ split imply

$$
\begin{aligned}
{ }^{3} R & =2 \partial_{\hat{1}} H_{\hat{1}}-H_{\hat{1}}{ }^{2}-H_{a b} H^{a b}+{ }^{2} R \\
h^{a b 3} R_{a b} & =\partial_{\hat{1}} H_{\hat{1}}-H_{a b} H^{a b}+{ }^{2} R \\
{ }^{2} D_{a} K_{\hat{1}}^{a} & =\partial_{\hat{1}} H_{\hat{0}}-H_{\hat{1}} H_{\hat{0}}+H_{\hat{1}} \operatorname{tr} K
\end{aligned}
$$

where ${ }^{2} R_{a b}$ is the Ricci 2-tensor of $h_{a b}$ and ${ }^{2} R=h^{a b} R_{a b}$.

We also need the timelike extrinsic 2-curvature of $S$ with respect to the timelike normal direction $\mathbf{e}_{\hat{0}}$; it is the 2 -tensor gotten by $2+1$ projection of $K_{i j}$,

$$
J_{a b}=\perp K_{a b}
$$

and the timelike mean extrinsic 2-curvature $H_{\hat{0}}$ is its 2-trace,

$$
H_{\hat{0}}=h^{a b} J_{a b}=h^{a b} K_{a b} ;
$$

here $\perp$ denotes the projection of 3-tensors into 2-tensors at $S$.

\section{Evolution of the Mean Extrinsic Curvatures}

We will present a system of equations which is fully covariant under transformations of the spatial coordinates $x^{i}$ and shift $\beta^{i}$ that preserve the inner-boundary constraints at $S$, Eqs. (9, 10). That is, we shall derive a system of equations which can be used with any choice of spatial gauge away from $S$. Some intermediate calculations, though, are most 
easily done in a particular spatial coordinate system, namely, Gaussian normal coordinates in space. Thus our spatial metric can be taken as

$$
\gamma_{i j} d x^{i} d x^{j}=d r^{2}+h_{a b} d x^{a} d x^{b}
$$

where $r \equiv x^{1}$. The inner boundary in spacetime is the hypersurface $r=r_{S}=$ const, consisting of all the $S$ for all $t$, which we want to be a spacelike or null hypersurface lying within or on the event horizon. Values at $S$ will be denoted " $\left.\right|_{S} "$. Thus by Eq. (10)

$$
\left.\beta_{\hat{1}}\right|_{S} \equiv B
$$

and also we will denote the inner boundary value the lapse at $S$ as

$$
\left.\alpha\right|_{S} \equiv A
$$

and its spatial normal derivative as

$$
\left.\partial_{\hat{1}} \alpha\right|_{S} \equiv A_{\hat{1}}
$$

At the inner boundary surface $S$, the two mean curvatures $\left.H_{\hat{0}}\right|_{S}$ and $\left.H_{\hat{1}}\right|_{S}$ are the quantities we wish to prescribe. Then we wish to derive these inner boundary values for the lapse $\alpha$ and shift $\beta^{i}$.

From these definitions and the Einstein equations follow the evolution equations for $H_{\hat{0}}$ and $H_{\hat{1}}$ :

$$
\begin{aligned}
\partial_{t}^{(n)} H_{\hat{0}}= & {\left[\frac{1}{2}\left(H_{\hat{0}}^{2}-H_{\hat{1}}^{2}\right)+\frac{1}{2}\left(H_{a b} H^{a b}+J_{a b} J^{a b}\right)-K_{r}^{a} K_{a}^{r}+8 \pi T_{\hat{0} \hat{0}}+\frac{1}{2}{ }^{2} R-{ }^{2} \Delta\right] A } \\
& +\left[J_{a b} H^{a b}+H_{\hat{0}} H_{\hat{1}}-H_{\hat{1}} \operatorname{tr} K-8 \pi T_{\hat{0} r}+\left({ }^{2} D_{a} K_{r}^{a}\right)+2 K_{r}^{a 2} D_{a}\right] B \\
& +\left[H_{\hat{1}}\right] A_{\hat{1}} \\
\partial_{t}^{(n)} H_{\hat{1}}= & {\left[J_{a b} H^{a b}-8 \pi T_{\hat{0} r}-\left({ }^{2} D_{a} K_{r}^{a}\right)-2 K_{r}^{a}{ }^{2} D_{a}\right] A } \\
& +\left[\frac{1}{2}\left(H_{\hat{0}}^{2}+H_{\hat{1}}^{2}\right)+\frac{1}{2}\left(H_{a b} H^{a b}+J_{a b} J^{a b}\right)+K_{r}^{a} K_{a}^{r}-H_{\hat{0}} \operatorname{tr} K\right. \\
& \left.+8 \pi T_{\hat{0} \hat{0}}-\frac{1}{2}{ }^{2} R+{ }^{2} \Delta\right] B \\
& +\left[H_{\hat{0}}\right] A_{\hat{1}}
\end{aligned}
$$

where $\partial_{t}^{(n)}$ denotes the projection of the time evolution operator $\partial_{t} \equiv \partial / \partial t$ normal to $S$,

$$
\partial_{t}^{(n)} \equiv A e_{\hat{0}}^{\mu} \frac{\partial}{\partial x^{\mu}}+B e_{\hat{1}}^{\mu} \frac{\partial}{\partial x^{\mu}}
$$

These equations are valid in any $3+1$ coordinate system that obeys Eqs. (9, 10) at $S$.

If we now view $H_{\hat{0}}$ and $H_{\hat{1}}$ as prescribed functions of $\left(t, x^{a}\right)$, Eqs. (18) become a set of two 2nd-order coupled partial differential equations in $\left\{x^{a}\right\}$ on $S$ for three unknown functions $A$, $B$ and $A_{\hat{1}}$. Thus, the inner boundary conditions for the lapse $\alpha$ and the radial component of the shift $\beta^{\hat{1}}$ are fixed. The tangential boundary value of the shift is still free, and can be chosen separately to enforce, say, "slip" or "no-slip" conditions as desired. 


\section{The Naturality of the Inner Boundary Conditions}

It may seem surprising that these equations involve $A_{\hat{1}}$ as well as $A$ and $B$; however a little reflection shows this to be natural and in fact desirable. To illustrate this, restrict to maximal slicing $\operatorname{tr} K=0$ temporarily. The maximal slicing equation

$$
0=\left({ }^{3} \Delta-K_{i j} K^{i j}-\text { matter }\right) \alpha
$$

is a 2 nd-order elliptic equation in $\left(x^{i}\right)$ for $\alpha$, and therefore admits at the inner boundary $S$ any of these well-posed boundary conditions:

$$
\begin{aligned}
\left.\alpha\right|_{S} & =A \quad \text { Dirichlet boundary conditions, or } \\
\left.\partial_{\hat{1}} \alpha\right|_{S} & =A_{\hat{1}} \quad \text { Neumann boundary conditions, or more generally, } \\
\left.F_{D} \alpha\right|_{S}+\left.F_{N} \partial_{\hat{1}} \alpha\right|_{S} & =0 \quad \text { homogeneous mixed boundary conditions; } \\
\left.F_{D} \alpha\right|_{S}+\left.F_{N} \partial_{\hat{1}} \alpha\right|_{S} & =F \quad \text { inhomogeneous mixed boundary conditions; }
\end{aligned}
$$

where $A, A_{\hat{1}}, F_{D}, F_{N}$, and $F$ are prescribed functions of $\left(t, x^{a}\right)$.

Conditions (21).21/d) simply say that there exists a prescribed linear relation between $\alpha$ and $\alpha_{\hat{1}}$ on $S$, but that neither is fixed individually; the distinction between these conditions (21),21d) is the function $F$ on the right-hand-side, which may be either 0 (homogeneous case) or a given function (inhomogeneous case).

Now, given $H_{\hat{0}}$ and $H_{\hat{1}}$, Eqs. (18) do give two prescribed linear relations among the three functions $(B-A),(B+A)$ and $A_{\hat{1}}$, albeit implicit ones. One can imagine using one of Eqs. (18) to eliminate $B$, whereupon the other becomes a single linear relation, in the form of Eq. (21 c), between $A$ and $A_{\hat{1}}$ - albeit an implicit one, involving Green functions of the operators $\mathcal{D}$ and $\mathcal{D}^{\prime}$. Thus, Eqs. (18) do appear to give well-posed boundary conditions for maximal slicing, Eq. (20), and they also should do so for attractive generalizations of maximal slicing [3].

In fact, not only is it admissible to have homogeneous mixed boundary conditions for the lapse $\alpha$ at $S$, it is also desirable. Imagine we instead used Dirichlet boundary conditions (17a). How big do we then make the inner boundary value $A$ ? - by which we mean, what fixes the overall scale of $A$ at the inner boundary? We would like to make it big enough so that, over a long evolution, the inner boundary just "keeps up" with the outer boundary, neither shooting way ahead, or falling way behind. How big is that? The first guess is $A=1$, but that cannot be right, because the inner boundary is in the strong field region. The lapse equation is not going to tell us how big to make $A$, precisely because it is happy with any $A$. Thus a pure Dirichlet boundary condition is not generally going to work well for long evolutions.

Thus, mixed homogeneous boundary conditions resolve this issue. They provide "boundary conditions without boundary values".

A homogeneous mixed boundary condition can work well - it can be thought of as a "feedback mechanism". If the inner boundary falls behind the outer boundary, then $A_{\hat{1}}$ will become large and positive, and the mixed boundary condition then can "tell" $A$ to become larger. If the inner boundary shoots ahead of the outer boundary, then $A_{\hat{1}}$ will become large and negative, and the mixed boundary condition then can "tell" $A$ to become smaller. A concrete example of this "mixed-boundary-condition feedback mechanism" will be presented 
below. Since the boundary condition is homogeneous, no data need be given at the inner boundary to fix the overall scale of $A$; this scale is self-adjusting.

\section{E. The PC Equations - Inner Boundary Conditions for Prescribed $\rho$ and $\rho^{\prime}$}

From Eqs. (10,18) can be found the evolution equations for the null convergences $\rho$ and $\rho^{\prime}$. When we take $\rho$ and $\rho^{\prime}$ as prescribed functions of $\left(t, x^{a}\right)$, these equations become the equations of prescribed convergence, or, for short, the PC equations:

$$
\begin{aligned}
\partial_{t}^{(n)} \rho= & \frac{1}{\sqrt{2}}\left[\rho\left(\rho^{\prime}+2 \rho-\operatorname{tr} K / \sqrt{2}\right)+\frac{1}{2} \mathcal{E}\right](B+A) \\
& +\frac{1}{\sqrt{2}}\left[\rho\left(-\rho^{\prime}+\rho-\operatorname{tr} K / \sqrt{2}\right)+\frac{1}{2} \mathcal{D}\right](B-A) \\
& +[\rho] A_{\hat{1}} \\
\partial_{t}^{(n)} \rho^{\prime}= & -\frac{1}{\sqrt{2}}\left[\rho^{\prime}\left(-\rho+\rho^{\prime}-\operatorname{tr} K / \sqrt{2}\right)+\frac{1}{2} \mathcal{D}^{\prime}\right](B+A) \\
& -\frac{1}{\sqrt{2}}\left[\rho^{\prime}\left(\rho+2 \rho^{\prime}-\operatorname{tr} K / \sqrt{2}\right)+\frac{1}{2} \mathcal{E}^{\prime}\right](B-A) \\
& -\left[\rho^{\prime}\right] A_{\hat{1}}
\end{aligned}
$$

a set of two $2 \mathrm{D}$ elliptic equations relating three unknown functions $A, B, A_{\hat{1}}$, where

$$
\begin{aligned}
\mathcal{D} & \equiv h^{a b}\left({ }^{2} D_{a}+K_{a}^{r}\right)\left({ }^{2} D_{b}+K_{b}^{r}\right)-\frac{1}{2}{ }^{2} R \\
\mathcal{D}^{\prime} & \equiv h^{a b}\left({ }^{2} D_{a}-K_{a}^{r}\right)\left({ }^{2} D_{b}-K_{b}^{r}\right)-\frac{1}{2}{ }^{2} R \\
\mathcal{E} & \equiv \frac{1}{2}\left(J_{a b}-H_{a b}\right)\left(J^{a b}-H^{a b}\right)-2 \rho^{2}+8 \pi\left(T_{\hat{0} \hat{o}}-T_{\hat{0} r}\right) \\
\mathcal{E}^{\prime} & \equiv \frac{1}{2}\left(J_{a b}+H_{a b}\right)\left(J^{a b}+H^{a b}\right)-2 \rho^{\prime 2}+8 \pi\left(T_{\hat{0} \hat{0}}+T_{\hat{0} r}\right)
\end{aligned}
$$

The latter quantities obey some useful relations. The two differential operators $\mathcal{D}$ and $\mathcal{D}^{\prime}$ live on $S$; they are not generally self-adjoint, due to the terms in $K_{a}^{r}$, but are the adjoints of each other:

$$
D^{\dagger}=D^{\prime}
$$

The two scalar functions $\mathcal{E}$ and $\mathcal{E}^{\prime}$ are nonnegative:

$$
\begin{aligned}
& \mathcal{E} \geq 0 \\
& \mathcal{E}^{\prime} \geq 0
\end{aligned}
$$

as long as matter obeys the dominant energy condition, which we will assume throughout. These equations are valid in any $3+1$ coordinate system that obeys Eqs. (9, 10) at $S$. 


\section{F. Special Case: Maximal Slices Bounded by Apparent Horizons}

As a special but important choice of coordinate conditions — still general enough to study binary black hole coalescences in $3+1 \mathrm{D}$ - let us take:

$$
\begin{aligned}
\operatorname{tr} K & =0 \quad \text { (maximal slicing) } \\
\rho & =0 \quad \text { (apparent-horizon inner boundary) } \\
\rho^{\prime} & =F\left(x^{a}\right) \quad \text { (some prescribed function) }
\end{aligned}
$$

Then the PC equations reduce to

$$
\begin{aligned}
& 0=\mathcal{E}(B+A)+\mathcal{D}(B-A) \\
& 0=\left(\mathcal{D}^{\prime}+2 \rho^{\prime 2}\right)(B+A)+\left(\mathcal{E}^{\prime}+4 \rho^{\prime 2}\right)(B-A)+2 \sqrt{2} \rho^{\prime} A_{\hat{1}}
\end{aligned}
$$

\section{G. Special Case: Stationary Black Holes}

Stationary black holes are relevant because calculations of binary coalescence will eventually settle down to a stationary black hole, and coordinate conditions are desirable that will somehow "lock onto" the geometry of the stationary black hole and render it recognizable. For a stationary black hole, we have

$$
\begin{aligned}
& \rho=0 \\
& \mathcal{E}=0
\end{aligned}
$$

and then from Eq. (23a) we have immediately on $S$

$$
B=A
$$

so that the $\mathrm{PC}$ conditions reduce to one $2 \mathrm{D}$ elliptic equation relating two unknown functions $A, A_{\hat{1}}$ :

$$
\partial_{t}^{(n)} \rho^{\prime}=-\sqrt{2}\left[\rho^{\prime}\left(\rho^{\prime}-\operatorname{tr} K / \sqrt{2}\right)+\frac{1}{2} \mathcal{D}^{\prime}\right] A-\left[\rho^{\prime}\right] A_{\hat{1}}
$$

The operator on $A$ is not obviously invertible; however in a later paper in this series, it will be proven that this operator is in fact invertable under fairly general conditions for a nonmaximal stationary black hole, and so the PC conditions can be expected to successfully "lock onto" the Kerr black hole at late times.

\section{CONCLUSION}

We have studied the apparent horizon boundary condition $\rho=0$ on a spacelike 2 -surface $S$ as an inner coordinate condition at a black hole. Since most apparent horizons are wild, another condition is required to ensure a well posed evolution. We propose the Prescribed Curvature Equations, or for short the PC Equations 


$$
\begin{array}{r}
\rho=(\text { some fixed function on } S) \\
\rho^{\prime}=(\text { some other fixed function on } S)
\end{array}
$$

an effective condition at the black hole. These equations have the following properties:

- They give rise to a system of two linear 2D elliptic equations on $S$ for three unknown boundary values of the lapse and shift.

- They therefore connect well with maximal slicing, and with related slicing conditions that involve 3D elliptic or parabolic equations.

- They are well posed and solvable for stationary black holes.

- They appear likely to be well posed and solvable under very general conditions.

Some additional numerical work will be required to solve the $\mathrm{PC}$ equations as part of a numerical relativity code. However, solving these equations cannot be not much harder than

finding apparent horizons in the first place, (see, e.g., 8.9]) and may well be easier because these equations are linear. Therefore the extra work seems unlikely to be prohibitive.

\section{ACKNOWLEDGMENTS}

This research was supported in part by the National Science Foundation under Grant Nos. PHY94-07194 and PHY90-08502 at ITP and UCSB. I am grateful for the hospitality of the the Texas/Los Alamos Workshop (IGPP, Feb. 1997), and grateful to workshop participants for many helpful comments on a version of this work. I am also grateful to Greg Cook, Sean Hayward, and Wai-Mo Suen for comments on the manuscript. 


\section{REFERENCES}

[1] M.A. Scheel, S.L. Shapiro, and S.A. Teukolsky, Collapse to black holes in Brans-Dicke theory. I. Horizon boundary conditions for dynamical spacetimes, gr-qc/9411025, Phys. Rev. D51, 4208 (1995).

[2] M.A. Scheel, S.L. Shapiro, and S.A. Teukolsky, Collapse to black holes in Brans-Dicke theory. II. Comparison with general relativity, gr-qc/9411026, Phys. Rev. D51, 4236 (1995).

[3] P. Anninos, G. Daues, J. Massó, E. Seidel, and W. Suen, Horizon boundary condition for black hole spacetimes, gr-qc/9412069, Phys. Rev. D51, 5562 (1995).

[4] E. Seidel and W. Suen, Towards a singularity-proof scheme in numerical relativity, Phys. Rev. Lett. 69, 1845 (1992).

[5] J. Balakrishna, G. Daues, E. Seidel, W. Suen, M. Tobias, and E. Wang Coordinate Conditions and Their Implementation in 3D Numerical Relativity, gr-qc/9601027, Class. Quant. Grav. 13, L135-L142 (1996).

[6] S.W. Hawking \& G.F.R. Ellis, The Large Scale Structure of Space-Time (Cambridge University Press, Cambridge, 1973) p. 320.

[7] R. Penrose and W. Rindler, Spinors and Space-Time. Vol. I (Cambridge University Press, Cambridge, 1984) Sect. 4.14.

[8] T.W. Baumgarte, G.B. Cook, M.A. Scheel, S.L. Shapiro, and S.A. Teukolsky, Implementing an apparent-horizon finder in three dimensions, gr-qc/9606010, Phys. Rev. D54, 4849 (1996).

[9] P. Anninos, K. Camarda, J. Libson, J. Massó, E. Seidel, and W. Suen Finding Apparent Horizons in Dynamic 3D Numerical Spacetimes, gr-qc/9609059, submitted to Phys. Rev. $D$. 